Case Report

\title{
Bradycardia, renal failure, shock, and hyperkalemia (BRASH) caused by AV nodal blocker: a case report of a patient with BRASH syndrome resistant to calcium administration
}

\author{
Sidhi Laksono ${ }^{1,2^{*}}$, Ananta Siddhi Prawara ${ }^{3}$ \\ 1) Department of cardiology and vascular medicine, RS Pusat Pertamina, South Jakarta, Indonesia \\ 2) Faculty of medicine, Universitas Muhammadiyah Prof. DR. Hamka, Tangerang, Indonesia \\ 3) Faculty of medicine, Universitas Diponegoro, Semarang, Indonesia
}

\section{A R T I C L E I N F O}

Submitted : $30^{\text {th }}$ August 2021 Accepted : $22^{\text {nd }}$ December 2021 Published : 25 $5^{\text {th }}$ January 2022

\section{Keywords:}

beta-blockers, bradyarrhythmia, bradycardia, hyperkalemia, renal failure, BRASH syndrome

\section{*Correspondence:}

sidhilaksono@uhamka.ac.id

This is an Open acces article under the CC-BY license

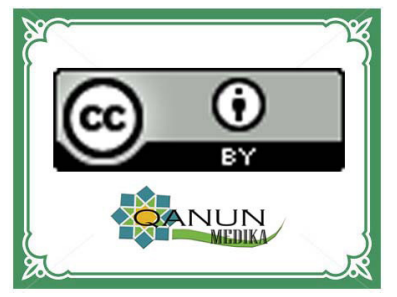

\begin{abstract}
Chronic kidney disease patients commonly present in a clinical setting with hypertension may cause or affect the disease. Carvedilol, a beta-blocker that is routinely used to treat hypertension in chronic kidney disease, was proven to be safer compared to other beta-blockers. However, it may still cause AV nodal block. AV nodal block can cause bradyarrhythmia, resulting in low cardiac output and low blood supply to multiple organs, including the kidney. This condition further impaired the kidney function in regulating potassium levels and cause hyperkalemia. Hyperkalemia, in return, can also cause bradycardia, and the vicious cycle goes on and on. Previous studies reported that calcium gluconate administration might significantly improve the patient's condition. However, in this case, calcium gluconate failed to give significant improvement even though it reduced the potassium level. We report a 53 years old male patient on carvedilol with bradyarrhythmia, chronic kidney disease, shock, and hyperkalemia (BRASH syndrome) that failed to resolve with calcium gluconate administration.
\end{abstract}




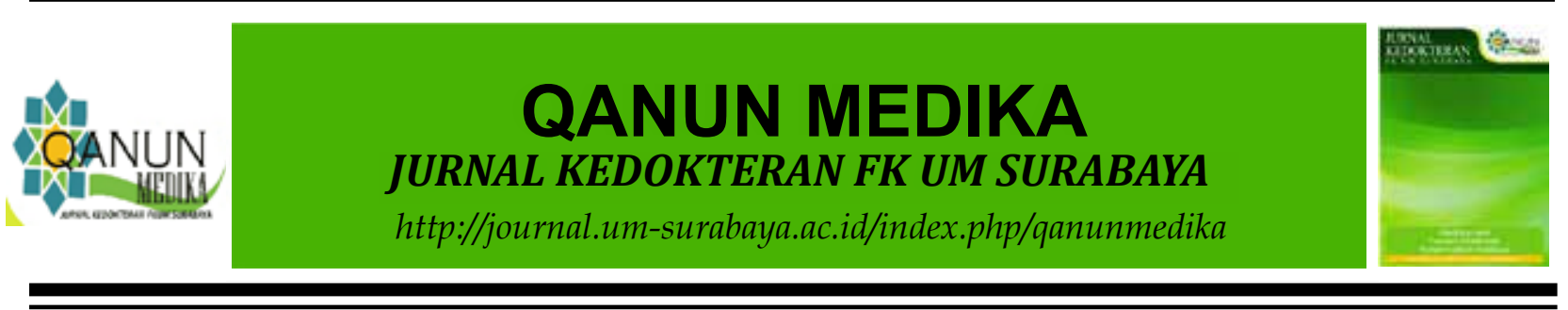

\section{INTRODUCTION}

Chronic kidney disease patient is common presented with hypertension in a clinical setting. Hypertension may act as both; the cause and effect of chronic kidney disease (Pugh, Gallacher, and Dhaun, 2020). Published guidelines suggested several drugs for hypertension control, one of which is beta-blocker. Compared to other betablockers, carvedilol is considered to be safer (Kidney Disease: Improving Global Outcomes (KDIGO) CKD Work Group, 2012). However, attention should be paid to the beta-blockers side effect, which is $\mathrm{AV}$ nodal block that causes bradycardia and low cardiac output. These conditions resulted in further impairment of renal function that provoked hyperkalemia because of impaired potassium clearance. Leaving these conditions unnoticed and untreated may be life-threatening (Bianchi et al., 2019). While managing these conditions with the bradycardia algorithm was ineffective (Farkas et al., 2020). We reported a case of 53 years old male on carvedilol for hypertension presented in the emergency room (ER) with junctional bradycardia, chronic kidney disease on hemodialysis, shock, and hyperkalemia (BRASH syndrome) that failed to resolve with calcium gluconate administration.

\section{CASE REPORT}

A 53 years old male was presented to the ER with fatigue. No dizziness or syncope episode was reported. The patient was previously diagnosed with chronic kidney disease and had been on routine dialysis. He was on carvedilol $6.25 \mathrm{mg}$ bid for hypertension and admitted that there was no change in the dosage. His pulse was $40 \mathrm{bpm}$, and blood pressure was 70/50 mmHg during admission. No abnormality was found during other physical examinations. The initial 12 lead electrocardiography (ECG) showed junctional rhythm with normal axis and $40 \mathrm{bpm}$ heart rate with normal QTc 405 ms (figure 1). The echocardiography showed a preserved ejection fraction of $55 \%$ with a low velocity-time integral of $15 \mathrm{~cm}$ and low cardiac output of 2.6 liters/minute. A routine swab test was done and showed a negative result for severe acute respiratory syndrome coronavirus 2 (SARS-CoV-2). Laboratory findings showed significant result in potassium $(6 \mathrm{mEq} / \mathrm{L}$, normal range $3.6-5.2 \mathrm{mEq} / \mathrm{L})$ and creatinine $(8.8 \mathrm{mg} /$ $\mathrm{dL}$, normal range $0.7-1.2 \mathrm{mg} / \mathrm{dL}$ ). The patient was diagnosed with BRASH syndrome. Two grams of calcium gluconate ( 2 attempts, 1 gram each attempt) were given as the initial treatment. The potassium decreased to $4.6 \mathrm{mEq} / \mathrm{L}$, but the patient remains bradycardia. Thus, dobutamine and epinephrine in combination were given to the patient. The patient was transferred to the intensive care unit to be closely monitored. However, the patient's condition deteriorated even after receiving the maximum dose of dobutamine and epinephrine. The patient was pronounced dead after four days of treatment in the hospital.

\section{DISCUSSION}

The incidence of BRASH syndrome is currently unknown because it is previously not reported as an entity. Patients with BRASH syndrome should be differentiated from other bradyarrhythmia patients because the two bradycardia cases' management is different (Farkas et al., 2020). In several studies, patients with BRASH syndrome reported dizziness, a history of syncope, weakness, difficulty breathing, and altered mental status as their chief complaint. In this case report, our patients only reported fatigue as his chief complaint. Our patient admits that he was taking carvedilol 6.25 $\mathrm{mg}$ bid for his hypertension without any dosage change. Thus, we can exclude intoxication with AV-nodal blocking agents, which is closely related to supratherapeutic drug levels. At the same time, BRASH syndrome occurred when there is a synergy between therapeutic drug levels and hyperkalemia (Farkas et al., 2020). 

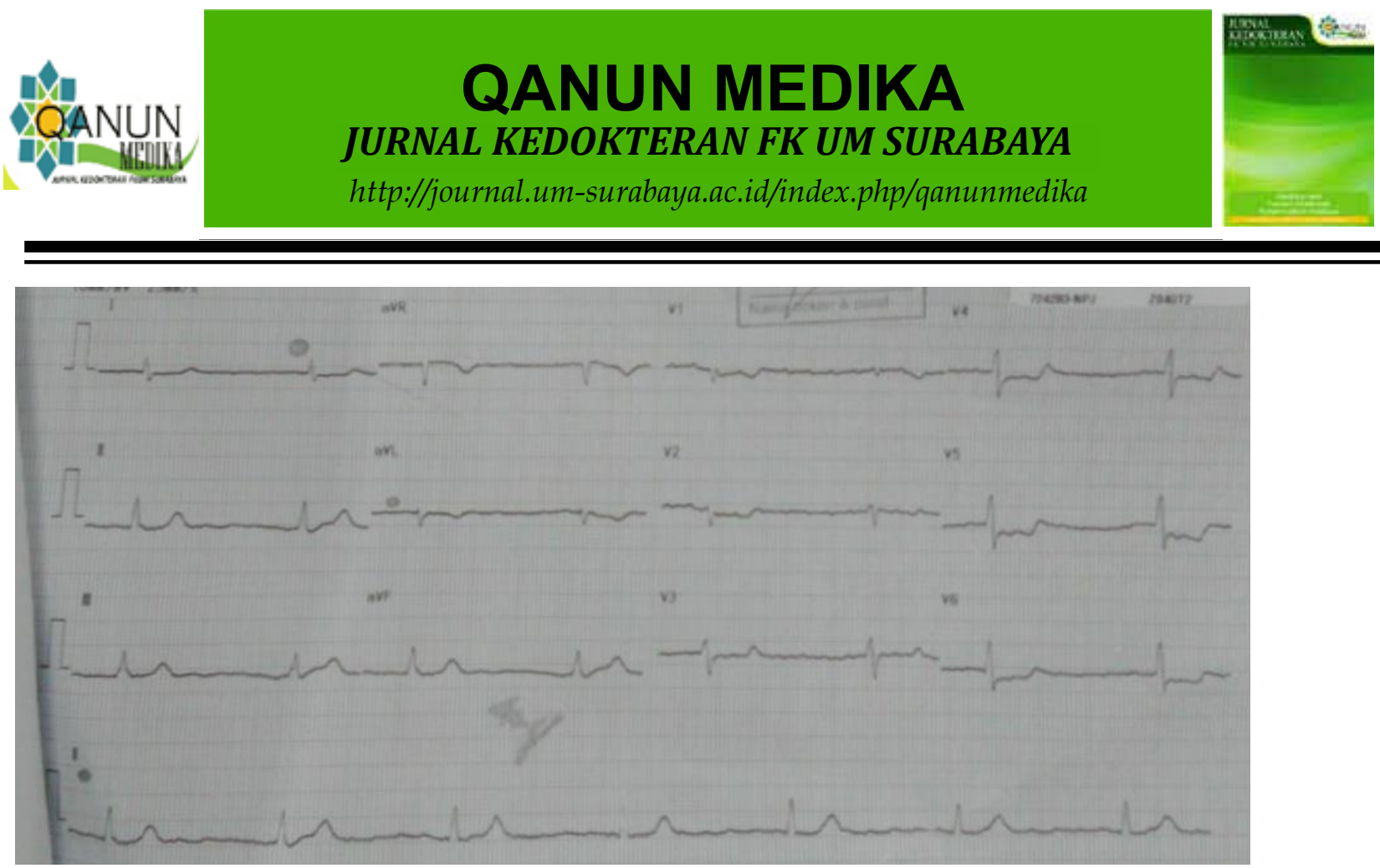

Figure 1. The 12 lead ECG of the patient during admission with junctional bradycardia

Carvedilol is a beta-blocker commonly used in managing hypertension, and it is reported to be safer than its beta-blocker predecessor. However, safer does not mean that it is free of side effects (Kidney Disease: Improving Global Outcomes (KDIGO) CKD Work Group, 2012). Its usage had also been reported in patients with BRASH syndrome in two previous studies, even though in both studies, carvedilol was not only consumed by the patients (Erden, Yalcin, and Ozhan, 2010; Aziz et al., 2011). Besides carvedilol, other drugs such as verapamil, amlodipine, trimethoprim-sulfamethoxazole, digitalis, Angiotensin-converting enzyme inhibitors (ACE-inhibitors), Angiotensin II receptor blockers (ARBs), and other betablockers (propranolol, metoprolol, atenolol, and sotalol) was also reported to be taken by BRASH syndrome patients (Farkas et al., 2020).

Hyperkalemia can occur with several mechanisms: 1) excessive intake, 2) impaired elimination of potassium, and 3) increased shift of potassium from intra to extracellular space (Lehnhardt and Kemper, 2011). Our patients did not report any additional drug taken besides carvedilol, no change in carvedilol dosage, and no high potassium intake. The cause of hyperkalemia in this patient is most likely associated with the elimination of potassium because he had chronic kidney disease on hemodialysis. Hyperkalemia further compounded bradyarrhythmia and low cardiac output caused by beta-blockers. Low cardiac output caused more impairment to the kidney function, and the vicious cycle continued (Schnaubelt et al., 2020).

The acute bradycardia management algorithm is widely known by healthcare providers, especially those who work in the emergency department. The acute bradycardia management algorithm suggested that the first step in treating bradycardia is by administering atropine sulfate, but the algorithm did not mention administering calcium gluconate (Kusumoto et al., 2019). Atropine sulfate was proven to be ineffective in managing bradyarrhythmia in BRASH syndrome patients. On the contrary, calcium gluconate was proven to give a significant clinical response to the patients (Farkas et al., 2020). Besides reducing potassium levels, calcium gluconate can stabilize the myocardium to increase heart rate, which correlates to more cardiac output (Farkas et al., 2020). We have managed our patients with two calcium gluconate administration 


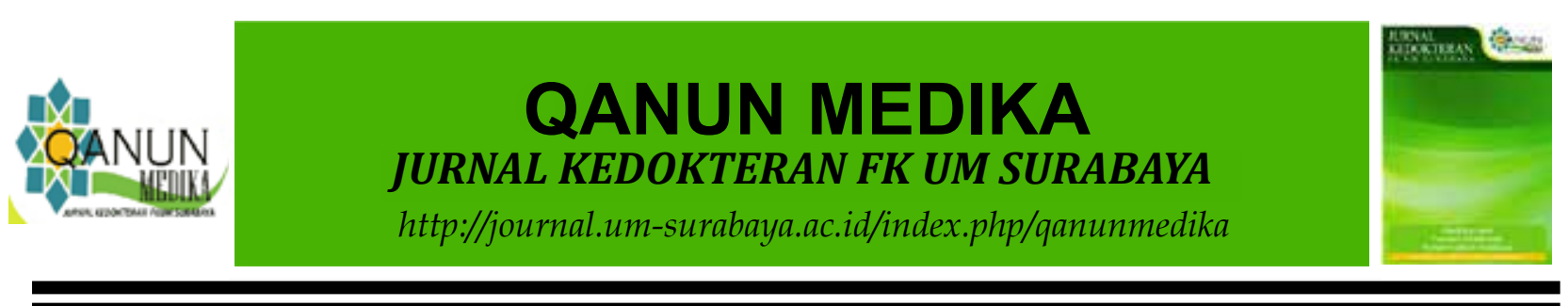

attempts (1 gram each), but the patient's clinical condition did not improve. Long B et al. recommended that calcium chloride via central access be administered after two failed calcium gluconate administration attempts. The next step is to enhance the redistribution of potassium by administering insulin and glucose, beta-agonists, and bicarbonate. However, in patients with chronic kidney disease on hemodialysis, only insulin administration could significantly lower potassium levels (Long et al., 2018). We did not administer calcium chloride via central access, insulin, and glucose in this patient because the potassium level has decreased to $4.6 \mathrm{mEq} / \mathrm{L}$.

Currently, no guideline specifically discusses the management of BRASH syndrome. However, a literature review based on several case reports concluded that BRASH syndrome management could be grouped into four major groups: potassium correction, fluid resuscitation, kaliuresis (with/without dialysis), and catecholamine for bradycardia and shock (Farkas et al., 2020).

Potassium corrections have been discussed previously. Fluid resuscitation was not done in this patient due to his previous medical condition (chronic kidney disease on hemodialysis). Hemodialysis should have been done in this patient because hemodialysis can help reduce potassium levels. However, we did not perform hemodialysis on this patient because the potassium levels have decreased after administering calcium gluconate. We have maximized our attempt in managing the bradycardia and shock using catecholamine with a combination of epinephrine and dobutamine and still failed to improve the patient's condition. The transvenous pacemaker was not necessarily used to managed BRASH syndrome patients (Farkas, 2016). Besides, the hospital facility was not possible to conduct transvenous pacemaker implantation, so a transvenous pacemaker was not used in this case. Furthermore, the referral was not performed because of the nontransportable condition of the patient.

\section{CONCLUSION}

Chronic kidney disease patients, especially on hemodialysis, should be monitored closely when prescribed, even with a single agent that can block AV nodal. In managing bradyarrhythmia patients, BRASHsyndromeshould beconsidered because it cannot be managed with the existing acute bradycardia management algorithm. Considering the complex situation of BRASH syndrome, which needs close monitoring and the possibility of needing hemodialysis and transcutaneous pacing, patients with BRASH syndrome could not be adequately managed in primary care settings. They should be referred as soon as possible to a complete facility, a hospital with a heart center is preferable. Calcium gluconate should be administered as the first-line treatment and can be repeated. The patient that failed to resolve the patient could be treated with calcium chloride via central access, insulin administration, hemodialysis, and catecholamine. A transvenous pacemaker was not necessarily used in managing BRASH syndrome patients but can be considered when all attempts have been made.

\section{REFERENCES}

Aziz, E. F. et al. (2011) 'Mild hyperkalemia and low eGFR a tedious recipe for cardiac disaster in the elderly : an unusual reversible cause of syncope and heart block', 6, pp. 41-44.

Bianchi, S. et al. (2019) 'Management of hyperkalemia in patients with kidney disease : a position paper endorsed by the Italian Society of Nephrology', Journal of Nephrology, 32(4), pp. 499-516.

Erden, I., Yalcin, S. and Ozhan, H. (2010) 'Syncope caused by hyperkalemia during 


\section{QANUN MEDIKA}

use of a combined therapy with the angiotensin-converting enzyme inhibitor and spironolactone', Kardiol Pol, 68(9), pp. 1043-1045.

Farkas, J. (2016) Bradycardia, Renal failure, AV blocker, Shock, and Hyperkalemia, Pulmcrit (EMCrit). Available at: https:// emcrit.org/pulmcrit/brash-syndromebradycardiarenal-failure-av-blockershock-hyperkalemia/ (Accessed: 8 October 2020).

Farkas, J. D. et al. (2020) 'BRASH Syndrome: Bradycardia, Renal Failure, AV Blockade, Shock, and Hyperkalemia', Journal of Emergency Medicine, 59(2), pp. 216-223.

Kidney Disease: Improving Global Outcomes (KDIGO) CKD Work Group (2012) 'KDIGO Clinical Practice Guideline for the Management of Blood Pressure in Chronic Kidney Disease', Kidney International, Supplement, 2, pp. 337414.
Kusumoto, F. M. et al. (2019) 'ACC / AHA / HRS GUIDELINE 2018 ACC / AHA / HRS Guideline on the Evaluation and Management of Patients With Bradycardia and Cardiac Conduction Delay', Circulation, pp. e382-e432.

Lehnhardt, A. and Kemper, M. J. (2011) 'Pathogenesis, diagnosis and management of hyperkalemia', pp. 377-384.

Long, B. et al. (2018) 'Controversies in Management of Hyperkalemia', Journal of Emergency Medicine. Elsevier Inc, (February), pp. 1-14.

Pugh, D., Gallacher, P. J. and Dhaun, N. (2020) 'Management of Hypertension in Chronic Kidney Disease', Drugs. Springer International Publishing, 79(4), pp. 365-379.

Schnaubelt, S. et al. (2020) 'The BRASH syndrome : an interaction of bradycardia , renal failure, AV block, shock and hyperkalemia', Internal and Emergency Medicine. pp. 2-4. 